\title{
Facilitated percutaneous coronary intervention
}

\section{B R Brodie}

Heart 2005;91:1527-1529. doi: 10.1136/hrt.2005.064618

$M$ ultiple, large randomised trials comparing primary percutaneous coronary intervention (PCI) with fibrinolytic therapy for ST elevation myocardial infarction (STEMI) have shown that primary PCI results in lower rates of death, reinfarction, and stroke. ${ }^{1}$ Consequently, primary PCI has become the preferred reperfusion strategy for STEMI. Unfortunately, primary PCI is available in only a minority of hospitals, and concern that treatment delays inherent in transporting patients from non-interventional hospitals to interventional hospitals may compromise outcomes, has limited the use of primary PCI. Recent trials in Europe have documented superior outcomes in patients with STEMI presenting at non-interventional hospitals when they are transferred to an interventional facility for primary PCI compared with being treated locally with fibrinolytic therapy, despite treatment delays of about one hour. ${ }^{23}$

Unfortunately, treatment delays in transferring patients from non-interventional to interventional hospitals are often longer than this in the "real world". This has stimulated interest in combining pharmacological treatment with mechanical reperfusion (facilitated PCI) in an attempt to minimise delays to reperfusion. Facilitated PCI is defined as the use of pharmacological treatment as soon as possible after the onset of STEMI in an attempt to establish early reperfusion, followed by transport to an interventional laboratory for emergent mechanical reperfusion in an attempt to maximise the frequency of TIMI 3 flow in the infarct artery and to stabilise the ruptured plaque with PCI. The facilitated PCI strategy triages all patients to the catheterisation laboratory for PCI following pharmacologic reperfusion therapy, and should be distinguished from rescue PCI, in which only patients who are thought to have unsuccessful reperfusion following pharmacologic reperfusion therapy are transported to the catheterisation laboratory for PCI.

\section{EVIDENCE SUGGESTING BENEFIT OF FACILITATED PCI}

Facilitated PCI potentially may improve outcomes over primary PCI alone by establishing earlier reperfusion, by providing an open artery on arrival to the catheterisation laboratory which may facilitate the PCI procedure, and by providing pharmacologic treatment which is synergistic with the PCI procedure. Two studies have documented that patients with STEMI who arrive at the catheterisation laboratory with an open versus a closed artery have superior outcomes. ${ }^{45}$ Our large database showed that patients with TIMI 2-3 flow ( $v$ TIMI $0-1$ flow) on initial angiography had better procedural results, fewer procedural complications, smaller infarct size, and lower in-hospital mortality. Recovery of left ventricular function and late cardiac survival were also better. The PAMI (primary angioplasty in myocardial infarction) investigators found that patients with TIMI 3 flow ( $v$ TIMI $0-2$ flow) on arrival at the catheterisation laboratory had better in-hospital outcomes and better survival at six months. ${ }^{5}$

\section{RESULTS OF EARLY STUDIES}

Early studies with facilitated PCI failed to show benefit when fibrinolytic therapy was combined with emergent percutaneous transluminal coronary angioplasty (PTCA). Most of these studies showed higher patency rates (TIMI 2-3 flow) on initial angiography in patients treated with fibrinolytic therapy, ${ }^{67}$ but none of these trials showed improved clinical outcomes, ${ }^{6-8}$ and major haemorrhagic complications were usually more frequent. ${ }^{78}$ The lack of benefit in these early trials may be related in part to the lack of synergy between fibrinolytic therapy and PTCA when performed without the benefit of platelet glycoprotein IIb/IIIa inhibitors and stents. Fibrinolytic therapy may not only result in increased bleeding when combined with invasive procedures but also may activate platelets and compromise the effectiveness of PTCA.

\section{RESULTS OF MORE RECENT STUDIES}

The introduction of stents and the use of platelet glycoprotein IIb/IIIa inhibitors, either alone or in combination with reduced dose fibrinolytic therapy, has allowed PCI to be performed more safely and synergistically following pharmacologic reperfusion therapy. A number of small pilot trials have evaluated facilitated PCI strategies using thrombolytic therapy, platelet glycoprotein IIb/IIIa inhibitors, or combinations of the two followed by emergent PCI.

The PACT (plasminogen-activator angioplasty compatibility trial) investigators documented more frequent TIMI 2-3 flow on initial angiography in patients treated with up-front half dose tissue plasminogen activator (tPA) followed by PCI versus primary PCI alone, but there were no differences in clinical outcomes or convalescent ejection fraction. ${ }^{9}$ The BRAVE (Bavarian reperfusion alternatives evaluation) investigators found more frequent TIMI 3 flow at initial angiography $(40 \% v 18 \%, \mathrm{p}<0.001)$ with half dose reteplase plus abciximab versus abciximab alone given before transfer to the catheterisation laboratory for emergent PCI, but they found no differences in clinical events or infarct size. ${ }^{10}$ The GRACIA-2 (grupo de analisis de la cardiopatia isquimica aguda) investigators found more frequent TIMI 3 flow at initial angiography (59\% $v 14 \%)$ and better ST segment resolution with up-front tenecteplase (TNK) versus placebo each followed by emergent PCI, but no differences in infarct size, left ventricular function, or clinical outcomes. ${ }^{11}$

Several studies have evaluated the effects of facilitation with up-front platelet glycoprotein IIb/IIIa inhibitors alone

Abbreviations: ASSENT- 4, assessment of the safety and efficacy of a new thrombolytic; BRAVE, Bavarian reperfusion alternatives evaluation; CAPTIM, comparison of angioplasty and pre-hospital thrombolysis in acute myocardial infarction; FINESSE, facilitated intervention with enhanced reperfusion speed to stop events; GRACIA, grupo de analisis de la cardiopatia isquimica aguda; $\mathrm{MI}$, myocardial infarction; ONTIME, ongoing tirofiban in myocardial infarction evaluation; PACT, plasminogen-activator angioplasty compatibility trial; $\mathrm{PCl}$, percutaneous coronary intervention; PTCA, percutaneous transluminal coronary angioplasty; STEMI, ST elevation myocardial infarction; TIMI, thrombolysis in myocardial infarction; TNK, tenecteplase; IPA, tissue plasminogen activator 
followed by emergent PCI. The ON-TIME (ongoing tirofiban in myocardial infarction evaluation) trial investigators found more frequent TIMI 2-3 flow at initial angiography in patients given up-front tirofiban versus tirofiban given in the catheterisation laboratory each followed by emergent PCI $(43 \% v 34 \%, p=0.04)$, but no differences in clinical events at one year. ${ }^{12}$ Montalescot and colleagues performed a metaanalysis of six trials comparing up-front abciximab with abciximab given in the catheterisation laboratory each followed by emergent PCI. ${ }^{13}$ Patients given up-front abciximab had a higher frequency of TIMI 2-3 flow at initial angiography $(42 \% \vee 30 \%, p<0.001)$, but there were only mild trends for improvement in clinical outcomes.

It appears clear from these pilot trials that the use of pharmacologic treatment up-front before emergent PCI will improve infarct artery patency at initial angiography, but larger trials are needed to evaluate clinical benefit with facilitated PCI.

\section{IMPORTANCE OF TIME TO TREATMENT WITH PRIMARY PCI}

The benefit of a facilitated PCI approach depends in part on the impact that treatment delays with primary PCI have on clinical outcomes. This has been a controversial subject, but observational data suggest that incremental treatment delays in performing primary PCI for STEMI which occur in the first $2-3$ hours after the onset of symptoms are critical and can greatly compromise outcomes. ${ }^{14}{ }^{15}$ After 2-3 hours, incremental treatment delays with primary PCI appear to have less effect on outcomes. This is probably related to the fact that there is a window of opportunity for myocardial salvage within the first two hours after the onset of symptoms, after which the opportunity for salvage is very modest. The benefits of very early reperfusion are related to myocardial salvage, and this is a very time dependent process. The benefits of later reperfusion are more related to the effects of an open artery in preventing remodelling and promoting electrical stability, and these effects are less time dependent.

Two clinical trials provide support for this paradigm. The PRAGUE-2 trial randomised patients with STEMI presenting at non-interventional hospitals to local fibrinolytic therapy versus transfer to interventional facilities for primary PCI. ${ }^{3}$ In patients randomised early $(<3$ hours), there was no difference in mortality between fibrinolytic therapy and transfer for PCI $(7.4 \% \vee 7.3 \%, \mathrm{p}=\mathrm{NS})$, while in patients randomised later ( $>3$ hours), there was a striking mortality advantage for primary PCI $(15.3 \% v 6.0 \%, \mathrm{p}=0.02)$. This can be explained in part by the fact that time delays in performing primary PCI early after the onset of symptoms greatly affect mortality, while incremental time delays later after the onset of symptoms have much less effect on mortality.

The CAPTIM (comparison of angioplasty and pre-hospital thrombolysis in acute myocardial infarction) trial randomised patients with STEMI to pre-hospital fibrinolytic therapy given by ambulance personnel in the home or workplace versus transfer for primary $\mathrm{PCI} .{ }^{16}$ In patients randomised early ( $<2$ hours), there was a strong trend for lower mortality with pre-hospital fibrinolytic therapy $(2.2 \% \mathrm{v}$ $5.7 \%, p=0.053)$, while in patients randomised later $(>2$ hours) there was no benefit to early fibrinolytic therapy, suggesting that time delays are very important early after the onset of symptoms but are less important later.

Thus, facilitated PCI is likely to be most effective in patients with STEMI who present early after the onset of symptoms and who can be treated early, and is likely to be less effective in patients presenting later after the onset of symptoms.

\section{CURRENT TRIALS AND CURRENT GUIDELINES}

Two large randomised trials are currently underway to evaluate the clinical benefit of facilitated PCI. The ASSENT4 (assessment of the safety and efficacy of a new thrombolytic) trial randomises patients with STEMI to TNK versus placebo each followed by emergent transfer for PCI. The FINESSE (facilitated intervention with enhanced reperfusion speed to stop events) trial randomises patients with STEMI to half dose reteplase plus abciximab versus abciximab versus placebo each followed by transfer for emergent PCI. The primary end point is a composite of death, heart failure, shock, or late ventricular fibrillation at 90 days. There is great hope and anticipation that the results of these trials will determine whether facilitated PCI is safe and effective and which patients will benefit most.

Until the results of these trials are available, the clinician must choose the best reperfusion strategy in patients with STEMI presenting at non-interventional hospitals based on an assessment of time and risk: (1) time from the onset of symptoms; (2) time delay in transport for primary PCI; (3) risk of STEMI; and (4) risk of fibrinolytic therapy. Patients who present early after the onset of symptoms $(<1-2$ hours $)$ who have expected long additional delays in transport for primary PCI $(>1$ hour) are probably best treated with thrombolytic therapy or combination therapy followed by emergent transport for PCI. Patients who present later after the onset of symptoms ( $>2$ hours) are probably best transported for primary PCI without fibrinolytic therapy, provided the transport times are not too long $(<2$ hours). These guidelines may need to be modified based on the risk of fibrinolytic therapy and whether the patient has a high risk STEMI. The major risk of fibrinolytic therapy is the risk of intracranial haemorrhage which occurs in about $1 \%$ of patients. The risk of major bleeding with combination therapy is about twice the risk with fibrinolytic therapy alone and includes the risk of intracranial haemorrhage in about $2 \%$ of patients $>75$ years old. ${ }^{17}$ Patients with low risk STEMI and high bleeding risk (for example, a 75 year old female with a small diaphragmatic myocardial infarction by ECG) should be considered for transfer for primary PCI without fibrinolytic therapy, even with early presentation. Patients with high risk STEMI and low bleeding risk (for example, a young man without hypertension and a large anterior myocardial infarction) should be considered for fibrinolytic therapy or combination therapy followed by transport for emergent PCI, even with later presentation. These guidelines are preliminary, and hopefully new guidelines can be developed and standardised when evidence from the large randomised trials becomes available.

Correspondence to: Dr Bruce R Brodie, Moses Cone Heart and Vascular Center, 313, Moses Cone Hospital, North Elm Street, Greensboro, NC 27408, USA; bbrodie@triad.rr.com

\section{REFERENCES}

1 Keeley EC, Boura JA, Grines CL. Primary angioplasty vs. intravenous thrombolytic therapy for acute myocardial infarction, a quantitative review of 23 randomized trials. Lancet 2003;361:13-20.

2 Andersen HR, Nielsen TT, Rasmussen K, et al. A comparison of coronary angioplasty with fibrinolytic therapy in acute myocardial infarction. N Engl J Med 2003;349(8):733-42.

3 Widimsky $P$, Budesinsky $T$, Vorac $D$, et al. Long distance transport for primary angioplasty vs immediate thrombolysis in acute myocardial infarction. Eur Heart J 2003;24:94-104.

4 Brodie BR, Stuckey TD, Hansen C, et al. Benefit of coronary reperfusion before intervention on outcomes after primary angioplasty for acute myocardial infarction. Am J Cardiol 2000;85:13-18.

5 Stone GW, Cox D, Garcia E, et al. Normal flow (TIMI-3) before mechanical reperfusion therapy is an independent determinant of survival in acute myocardial infarction: analysis from the primary angioplasty in myocardial infarction trials. Circulation 2001;104:636-41.

6 Vermeer F, Oude Ophuis AJ, vd Berg EJ, et al. Prospective randomized comparison between thrombolysis, rescue PTCA, and primary PTCA in 
patients with extensive myocardial infarction admitted to a hospital without PTCA facilities: a safety and feasibility study. Heart 1999;82:426-31.

7 Widimsky P, Groch L, Zelizko M, et al. Multi-center randomized trial comparing transport to primary angioplasty vs. immediate thrombolysis vs. combined strategy for patients with acute myocardial infraction presenting to a community hospital without a catheterization laboratory. Eur Heart $J$ 2000;21:823-31

8 O'Neill WW, Weintraub R, Grines CL, et al. A prospective, placebocontrolled, randomized trial of intravenous streptokinase and angioplasty versus lone angioplasty therapy of acute myocardial infarction. Circulation 1992:86:1710-17.

9 Ross AM, Coyne KS, Reiner JS, et al. A randomized trial comparing primary angioplasty with a strategy of short-acting thrombolysis and immediate planned rescue angioplasty in acute myocardial infarction: the PACT trial. J Am Coll Cardiol 1999:34:1954-62.

10 Kastrati A, Mehilli J, Schlotterbeck K, et al. Early administration of reteplase plus abciximab vs abciximab alone in patients with acute myocardial infarction referred for percutaneous coronary intervention: a randomized controlled trial. JAMA 2004;291:947-54.

11 Fernandez-Aviles F, Alonso JJ, Castro-Beiras A, ef al. Primary angioplasty versus facilitated intervention (tenecteplase plus stenting) in patients with STelevation acute myocardial infarction: final results of the GRACIA-2 trial. J Am Coll Cardiol 2004;43(suppl A):289A (abstract).
12 van't Hof AW, Ernst N, de Boer MJ, et al. Facilitation of primary coronary angioplasty by early start of a glycoprotein $\mathrm{Ilb} / \mathrm{Illa}$ inhibitor: results of the ongoing tirofiban in myocardial infarction evaluation (On-TIME) trial. Eur Heart J 2004;25:837-46.

13 Montalescot G Borentain M, Payot L, et al. Early vs late administration of glycoprotein $\mathrm{llb} / \mathrm{llla}$ inhibitors in primary percutaneous coronary intervention of acute ST-segment elevation myocardial infarction: a meta-analysis. JAMA 2004; 292:362-6

14 Brodie BR, Stuckey TD, Wall TC, et al. Importance of time to reperfusion for 30-day and late survival and recovery of left ventricular function after primary angioplasty for acute myocardial infarction. J Am Coll Cardiol 1998;32:1312-19.

15 Brodie BR, Cox DA, Stuckey TD, et al. How important is time to treatment with primary percutaneous coronary intervention for acute myocardial infarction? Results from the CADILAC trial. J Am Coll Cardiol 2003;41(suppl 5):368A (abstract).

16 Steg PG, Bonnefoy E, Chabaud S, et al. Impact of time to treatment on mortality after prehospital fibrinolysis or primary angioplasty: data from the CAPTIM randomized clinical trial. Circulation 2003;108:2851-6.

17 The GUSTO V Investigators. Reperfusion therapy for acute myocardial infarction with fibrinolytic therapy or combination reduced fibrinolytic therapy and platelet glycoprotein Ilb/Illa inhibition: the GUSTO V randomized trial. Lancet 2001;357:1905-14.

\section{IMAGES IN CARDIOLOGY}

\section{Siblings with supravalvar aortic stenosis}

$T^{T}$ wo brothers were referred to a tertiary care hospital for investigation of cardiac murmurs. The elder brother was 8 years old, and was asymptomatic. Examination revealed normal blood pressure in both upper limbs and a normal sized heart with an ejection systolic murmur best heard in the right second space radiating to the right carotid artery. The ECG was essentially normal but echocardiography revealed a gradient of $70 \mathrm{~mm} \mathrm{Hg}$ at the supravalvar level. Diagnostic angiography revealed discrete supravalvar stenosis with a gradient of $45 \mathrm{~mm} \mathrm{Hg}$ (panels A and B) Coronary and pulmonary arteries were normal. The younger brother was 6 years old and complained of angina on exertional activity. His clinical examination revealed normal blood pressure in both the upper extremities and a sustained left ventricular apex. There was an ejection systolic murmur best heard in the right second space radiating to the right carotid artery. His ECG revealed left ventricular hypertrophy and echocardiography revealed a diffuse narrowing of the ascending aorta and a gradient of $200 \mathrm{~mm} \mathrm{Hg}$ across it. Diffusely dilated coronary arteries were seen. Diagnostic angiography revealed diffuse narrowing of the supravalvar aorta, and ostial stenosis of the left common carotid artery with diffuse dilatation of the left coronary system (panels C and D) Pulmonary artery angiography did not reveal pulmonary artery stenosis.

Both brothers had facial dysmorphism but they did not fit into any syndrome. There was no family history and the eldest brother of the two boys was normal upon cardiac investigation. Their serum calcium concentrations were normal. Both brothers are awaiting surgical repair.

\section{S Deshpande \\ S Khadse \\ V Bhatia \\ drshantanud@yahoo.com}
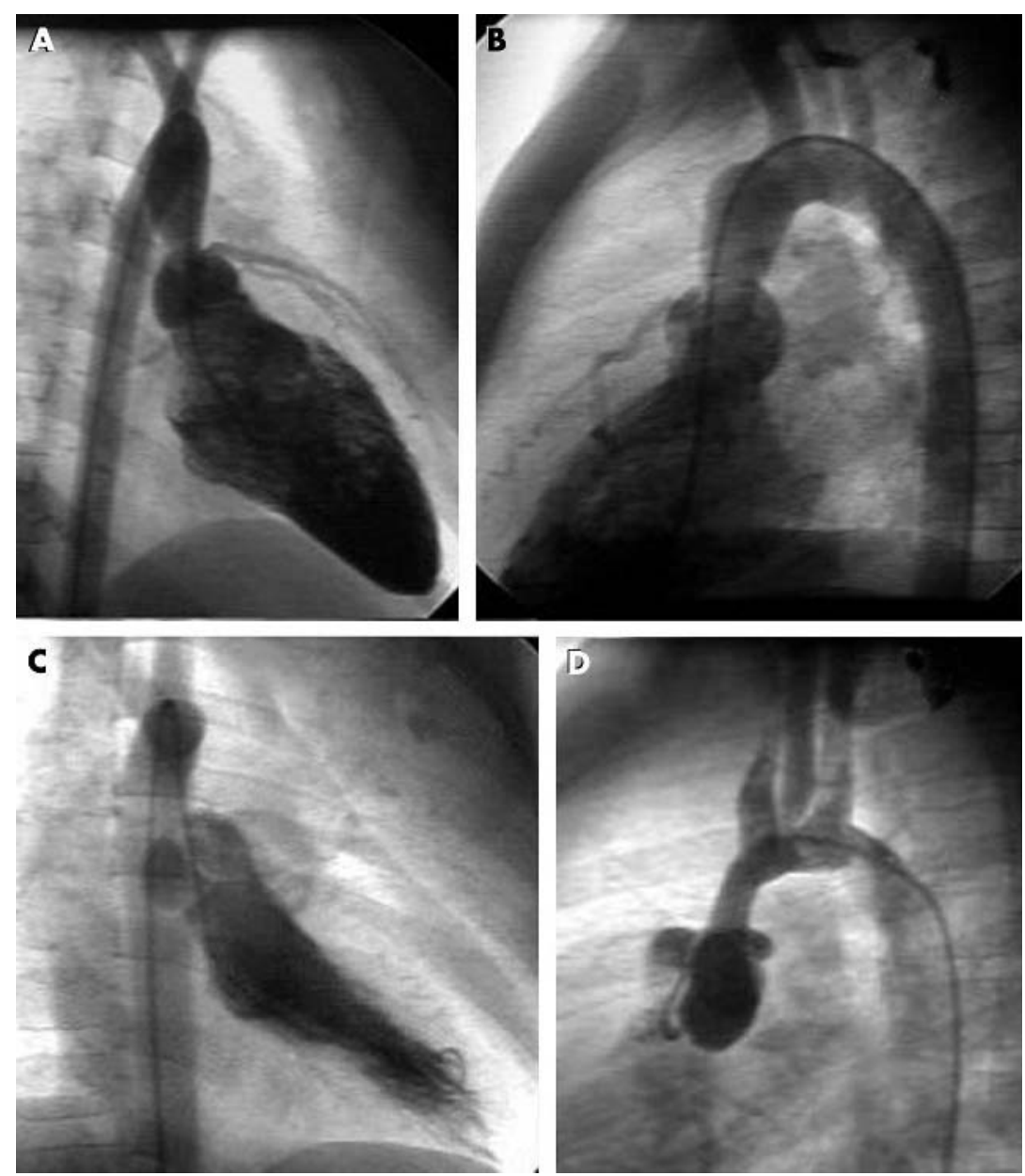